\section{OP05 EVALUATING SOCIAL AND SPATIAL INEQUALITIES OF LARGE SCALE RAPID LATERAL FLOW SARS-COV-2 ANTIGEN TESTING IN COVID-19 MANAGEMENT: AN OBSERVATIONAL STUDY OF LIVERPOOL, UK (NOVEMBER 2020 TO JANUARY 2021)}

${ }^{1}$ Mark Green*, ${ }^{2}$ Marta Garcia-Finana, ${ }^{3}$ Ben Barr, ${ }^{2}$ Girvan Burnside, ${ }^{2}$ Chris Cheyne, ${ }^{2}$ David Hughes, ${ }^{4}$ Matthew Ashton, ${ }^{3}$ Sally Sheard, ${ }^{3}$ Iain Buchan. ' Geography and Planning, University of Liverpool, Liverpool, UK; ${ }^{2}$ Health Data Science, University of Liverpool, Liverpool, UK; ${ }^{3}$ Public Health and Policy, University of Liverpool, Liverpool, UK; ${ }^{4}$ Public Health, Liverpool City Council, Liverpool, UK

\subsection{6/jech-2021-SSMabstracts.5}

Background Asymptomatic transmission of SARS-CoV-2 poses a significant burden on managing the spread of COVID-19. Few studies have evaluated the impact of testing for asymptomatic COVID-19 among large populations or whole cities using empirical data. No study to our knowledge has considered if such interventions result in or exacerbate existing socioeconomic inequalities. The aim of our study is to explore social and spatial inequalities in uptake and case-detection of rapid lateral flow SARS-CoV-2 antigen tests (LFTs) offered to people without symptoms of COVID-19 in Liverpool between 6th November 2020 and 31st January 2021.

Methods Linked pseudonymised records for asymptomatic residents in Liverpool (UK) who received a LFT for COVID-19 between 6th November 2020 to 31st January 2021 were accessed using the Combined Intelligence for Population Health Action (CIPHA) data resource. Bayesian Hierarchical Poisson Besag, York, and Mollié models were used to estimate ecological associations for uptake and positivity of testing.

Results 214525 residents (43\%) received a LFT identifying 5557 individuals as positive cases of COVID-19 (1.3\%). Uptake was highest in November when there was military assistance. High uptake was observed again in the week preceding Christmas and was sustained into a national lockdown. Overall uptake and repeat testing were lower among males (e.g. $40 \%$ uptake over the whole period), Black Asian and other Minority Ethnic groups (e.g. 27\% uptake for 'Mixed' ethnicity) and in the most deprived areas (e.g. 32\% uptake in most deprived areas). These population groups were also more likely to have received positive tests for COVID-19. Models demonstrated that uptake and repeat testing were lower in areas of higher deprivation, areas located further from test sites and areas containing populations less confident in the using Internet technologies. Positive tests were spatially clustered in deprived areas.

Conclusion Our study provides the first substantial evidence on inequalities involved in large-scale asymptomatic rapid testing of populations for SARS-CoV-2. Large-scale voluntary asymptomatic community testing saw social, ethnic, and spatial inequalities in an 'inverse care' pattern, but with an added digital exclusion factor. While test uptake was popular, there was a disconnect between the populations accessing testing and those experiencing harms relating to COVID-19. COVID19 testing and support to isolate need to be more accessible to the vulnerable communities most impacted by the pandemic, including non-digital means of access.

\section{OP06 DIFFERENCES IN THE RATES OF NEW DIAGNOSES REPORTED PRE AND DURING THE COVID-19 PANDEMIC}

Jennifer Cole*, Shivan Thakrar, Helen M Parretti, Nick Steel. Department of Medicine, University of East Anglia, Norwich, UK

10.1136/jech-2021-SSMabstracts. 6
Background Excess deaths from conditions other than COVID-19 have been reported during the pandemic. Difficulty accessing healthcare is a possible explanation. We aimed to assess changes in rates of new diagnoses for common conditions before and during the pandemic.

Methods Participants aged 50 years and older in three waves of the English Longitudinal Study for Ageing were asked whether a doctor had told them that they had newly developed any of 11 medical conditions: hypertension, angina or heart attack, heart failure, diabetes, stroke, chronic lung disease, asthma, arthritis, cancer, dementia, or blood disorder. Responses were compared between pre-COVID Wave 9 (W9) in 2018 to 2019, COVID Wave 1 (CW1) in June and July 2020, and COVID Wave 2 (CW2) in November and December 2020. Multivariate logistic regression on weighted data was performed to assess variation by sex, age group (50 to state pension age (SPA), SPA to 74,75 and over), and wealth quintile.

Results The overall rate of new diagnoses reported per 1,000 participants per year was 116.76 in W9 $(n=7,289), 110.10$ in CW1 $(\mathrm{n}=5,825)$, and 138.23 in CW2 $(\mathrm{n}=5,339)$. Diagnosis rates dropped from W9 to CW1 to CW2 for dementia (9.09 to 4.01 to 2.25 respectively), angina and heart attack (13.50 to 11.67 to 10.86 ), and stroke (7.05 to 6.18 to 4.12 ). In contrast, the rate increased for arthritis (29.11 to 37.42 to 44.58 ) and diabetes (18.83 to 21.06 to 23.60 ). Multivariate analysis found that increasing age was a predictor for dementia diagnosis in cw2 (odds ratio 2.20; 95\% confidence interval 1.12 to 4.30). No other regression analyses were statistically significant.

Conclusion Drops in diagnosis rates for dementia and cardiovascular disease during the pandemic could be contributing to increased deaths from conditions other than COVID-19. Policy initiatives are underway to encourage people to seek healthcare as normal, and it will be important to maintain adequate access to healthcare for major conditions in any future lockdown. The reasons for the reported substantial rise in arthritis and smaller rise in diabetes diagnosis rates are not clear and require further investigation.

Wednesday 15 September

CVD \& CVD Modelling, $13.00-15.25$

\section{OP07 ASSOCIATION BETWEEN CARDIOVASCULAR HEALTH AND HEART FAILURE IN OLDER BRITISH MEN; FINDINGS FROM THE BRITISH REGIONAL HEART STUDY}

${ }^{1}$ Mehar Chawla*, 'Olia Papacosta, 'Lucy Lennon, ${ }^{2}$ Peter Whincup, 'S Goya Wannamethee. ${ }^{1}$ Department of Primary Care and Population Health, University College London, London, UK; ${ }^{2}$ Population Health Research Institute, St George's University of London, London, UK

\subsection{6/jech-2021-SSMabstracts.7}

Background With an ageing population heart failure (HF) is emerging as a common cardiovascular condition and is one of the leading causes of mortality and morbidity. The American Heart Association promotes Life simple's 7 (LS7) to improve cardiovascular health (CVH). LS7 consists of seven modifiable health factors: body mass index, blood pressure, glucose, cholesterol, physical activity, smoking and diet. We sought to examine the impact of LS7 on risk of incident HF in older British men as well as the overall 
CVH trajectory from middle age to older age and subsequent HF risk.

Methods In 1998-2000, 20 years after the initial screening of 7735 men aged 40-59 years (The British Regional Heart Study), 4252 men were re-examined when aged 60-79 years, with collection of all LS7 components and follow-up for a median period of 15.8 years. Men with previous history of cardiovascular disease were excluded leaving 3698 men. The composite LS7 score ranges from $0-14$ and men were classified as having poor (1-4), intermediate (5-10) and ideal (1114) $\mathrm{CVH}$ score. Four CVH trajectory groups were created based on transition between low and high $\mathrm{CVH}$ score from middle to older age:(1) Low-Low, (2) Low-High, (3), HighLow, and (4) High-High. Cox models were used to estimate the risk of HF adjusted for age, alcohol consumption, socioeconomic class and incident myocardial infarction.

Results Of the 3698 men 14\% $(n=522)$ had ideal CVH score. Ideal $\mathrm{CVH}$ was associated with a significant decrease in risk of $\mathrm{HF}$ compared to those with a poor $\mathrm{CVH}$ score (HR 0.52, $95 \% 0.31$ to $0.88, \mathrm{p}<0.016)$; intermediate $\mathrm{CVH}$ was associated with reduced but non-significant $\mathrm{HF}$ risk (HR 0.79, 95\% CI 0.51 to $1.24, \mathrm{p}=0.310$ ). Compared to the Low-Low CVH trajectory group, those who maintained a healthy $\mathrm{CVH}$ score (High-High) showed the lowest risk of HF (HR 0.67, 95\% CI 0.51 to $0.87, \mathrm{p}<0.003)$; those who moved from high to low showed lower but non-significant risk (HR 0.78 95\% CI 0.55 to $1.11, \mathrm{p}=0.17)$; those who moved from low to high showed no benefit (HR $1.0195 \%$ CI 0.76 to 1.33).

Discussion Our findings suggest that having ideal $\mathrm{CVH}$ reduces the risk of developing HF in older age. LS7 is a simple way to identify high risk individuals however the prevalence of older men with ideal $\mathrm{CVH}$ is low. Adopting and maintaining healthy cardiovascular health from middle age to older age confers the most benefit in preventing $\mathrm{HF}$ in later life.

\section{OP08 SOCIOECONOMIC POSITION AND SEX-SPECIFIC TRAJECTORIES OF METABOLITES FROM CHILDHOOD TO EARLY ADULTHOOD: A PROSPECTIVE COHORT STUDY}

${ }^{1}$ Kate O'Neill*, 1,2,3 Linda O'Keeffe. 'School of Public Health, University College Cork, Cork, Ireland; ${ }^{2}$ MRC Integrative Epidemiology Unit, University of Bristol, Bristol, UK; ${ }^{3}$ Population Health Sciences, Bristol Medical School, Bristol, UK

\subsection{6/jech-2021-SSMabstracts.8}

Background Socioeconomic inequalities in cardiovascular disease are stronger and more consistent in females compared with males. However, mechanisms underlying these inequalities and whether they emerge in early life are unclear.

Methods Trajectories of 148 metabolic trait concentrations from age $7 \mathrm{y}$ to $25 \mathrm{y}$ in a contemporary English birth cohort, The Avon Longitudinal Study of Parents and Children (ALSPAC), were analysed. Outcomes included concentrations of metabolic traits quantified using nuclear magnetic resonance spectroscopy measured at 7y,15y, 18y and 25y. Maternal education was used as an indicator of socioeconomic position (SEP), reported by mothers at 32-weeks gestation. Using linear spline multilevel models, sex-specific associations of SEP and trajectories of each metabolic trait concentration were examined. Sex-specific associations were converted to standard deviation (SD) units by dividing the predicted total absolute difference from $7 \mathrm{y}$ to $25 \mathrm{y}$ by SEP in original units by the sex-specific SD of the trait in the reference SEP category (degree level maternal education).

Results Total participants included ranged from 5,980-6,212 with 10,023-11,945 repeated measures. SEP was associated with numerous metabolic traits trajectories in females, some which developed or strengthened by age $25 \mathrm{y}$ with evidence of an emerging SEP gradient. Associations were strongest for large HDL and VLDL cholesterol, apolipoprotein B/A-1 and glycoprotein acetyls. For instance, by age 25y less than O-level education was associated with 0.37 SD (95\% CI: 0.21, 0.5.3), O-level with 0.28 SD (95\% CI: 0.14, 0.42) and A-level with 0.08 SD (95\% CI: -0.6, 0.22) higher concentrations of glycoprotein acetyls compared to degree level education. In males, associations between SEP and metabolic traits were weaker and less consistent with little evidence of an SEP gradient.

Conclusion Sex differences in socioeconomic inequalities in cardiometabolic risk appear to develop early in the life course and are evident by early adulthood with more adverse effects of SEP in females.

\section{OP09 QUANTIFYING BENEFITS OF THE DANISH TRANSFAT BAN FOR CORONARY HEART DISEASE MORTALITY 1991-2007: SOCIOECONOMIC ANALYSIS USING THE IMPACT $_{S E C}$ MODEL}

${ }^{1}$ Kirsten Schroll Bjoernsbo*, ${ }^{2}$ Albert Marni Joense, ${ }^{1,3,4}$ Torben Joergensen, ${ }^{5}$ Søren LundbyeChristensen, ${ }^{6}$ Anette Bysted, ${ }^{6}$ Tue Christensen, ${ }^{7}$ Simon Capewell, ${ }^{7}$ Martin O'Flaherty. ${ }^{1}$ Center for Clinical Research and Prevention, Bispebjerg and Frederiksberg Hospital, Frederiksberg, Denmark; ${ }^{2}$ Aalborg University Hospital, Department of Cardiology, Aalborg, Denmark; ${ }^{3}$ Department of Public Health, Faculty of Health and Medical Sciences, University of Copenhagen, Copenhagen Denmark; ${ }^{4}$ Faculty of Medicine, Aalborg University, Aalborg, Denmark; ${ }^{5}$ Unit of Clinical Biostatistics, Aalborg University Hospital, Aalborg, Denmark; ${ }^{6}$ National Food Institute, Technical University of Denmark, Kgs. Lyngby, Denmark; ${ }^{7}$ University of Liverpool, Department of Public Health and Policy, Liverpool, UK

\subsection{6/jech-2021-SSMabstracts.9}

Background In 2004, the Danish Transfat Ban was implemented (Order no. 160, 2003). To determine health and equity benefits of this regulation on industrially produced trans fatty acid (ITFA) content in Danish food, we quantified the changes in ITFA intake from 1991 to 2007. We then estimated the relative contributions of changes in ITFA consumption, other cardiovascular risk factors and treatments on Coronary Heart Disease (CHD) mortality, all stratified by socioeconomic group.

Methods Participants included adult Danes aged 25-84 years living in Denmark in 1991 and 2007, stratified by socioeconomic quintiles. Population counts were obtained from the Danish Central Office of Civil Registration, and financial income data from Statistics Denmark. ITFA intake data as grams/day and $\%$ total energy intake $(\% \mathrm{E})$ were obtained from dietary assessments from the DanMonica 3 Study (1991) and the Danish National Surveys of Dietary Habits and Physical Activity (DANSDA, 2005-2007) combined with estimated ITFA content in foods on the Danish market. We extended the previously validated Danish IMPACT $_{\mathrm{SEC}}$ model to quantify reductions in CHD mortality; the principal output being the number of CHD deaths prevented or postponed attributable to changes in ITFA (\%E) intake between 1991 - 2007, all stratified by socioeconomic group. To quantify degrees of uncertainty, we conducted robust sensitivity analyses.

Results Between 1991 and 2007, mean ITFA intake in Denmark fell dramatically from $2.9 \mathrm{~g} / \mathrm{d}$ to $0.3 \mathrm{~g} / \mathrm{d}$ in men, and 